\title{
Constrained filtering method for attitude determination using GPS and gyro
}

\author{
Y.T. Chiang, L.S. Wang, F.R. Chang and H.M. Peng
}

\begin{abstract}
In attitude determination using the unit quaternion representation, the unit norm constraint results in singularity of the covariance matrix using classical filtering methods. To cope with this problem, a constrained filtering method is developed. In this method, the constraint equations and the state equations are treated as pseudo-measurement equations, so that they can be processed simultaneously with the real measurement equations. To illustrate the application of the proposed scheme, a scenario using GPS and gyro to perform attitude determination is considered. Software simulations show that the proposed scheme is sound, effective, and superior to existing methods.
\end{abstract}

\section{Introduction}

The problem in attitude determination is to find a special orthogonal matrix that transforms the coordinates between the reference frame and the body frame. Among the various representations, the unit quaternion is appropriate when dealing with large angle manoeuvres. However, as shown in [1], the problem of a singular covariance matrix arises inevitably when applying the classical Kalman filtering algorithm. This is due to the lack of independency of the four quaternion components, which are related by the constraint that the quaternion has a unit norm. This constraint is difficult to maintain numerically in the presence of accumulated round-off errors, which may even give rise to a negative-definite covariance matrix. To cope with this problem, some reduction methods, either using a reduced covariance matrix or using a reduced state equation, have been proposed in [1]. The constraint condition is handled indirectly in these methods, and hence the filters designed are more susceptible to initial conditions. It is desirable to develop a filtering method which not only solves the singularity problem directly but also converges rapidly for arbitrary initial conditions. The constrained filtering method proposed in this paper provides a candidate to suit such needs.

The proposed scheme is a recursive estimation method that can deal with system equations, measurement equations and constraint equations simultaneously, linear or nonlinear. The idea is to treat both system equations and

(C) IEE, 2002

IEE Proceedings online no. 20020531

DOI: 10.1049/ip-rsn:20020531

Paper first received 10th January and in revised form 23rd May 2002

Y.T. Chiang and F.R. Chang are with the Department of Electrical Engineering, National Taiwan University, 1 Section 4 Roosevelt Road, Taipei, Taiwan 106, Republic of China

L.S. Wang is with the Institute of Applied Mechanics, National Taiwan University

H.M. Peng is with the Advance Technology Rescarch Lab., Chung Hwa Telecommunication Laboratorics, 12, Lane 551, Min-Tsu Road Sec. 5, Yang-Mci, Taoyuan, Taiwan 326, Republic of China

258 constraint equations as pseudo-measurement equations. The corresponding equations from the former may be corrupted by process noise, while those from the latter have zero covariance matrix. The error covariance matrix of the converted system then becomes singular, and the conventional maximum likelihood estimation algorithm needs to be modified. The concept has been discussed in [2] to overcome the filtering problem for linear descriptor systems, for which the estimation scheme is derived by applying Lagrange multiplier techniques. This method was used in [3] to carry out attitude determination using GPS measurements with linearised equations and linearised constraint conditions. In this paper, the method is extended to solve the singularity problem associated with the nonlinear constraint of unit quaternion in attitude determination.

To illustrate the proposed method, a scenario using GPS and gyro to perform attitude determination is considered $[4,5]$. On a platform consisting of two baseline vectors, three non-co-linear antennas arc used to acquire GPS satellite signals, and the Litton SIRU gyro is chosen to measure angular rates. The performance of the fusing algorithm developed in this paper is verified by software simulations. The results show that the proposed filtering method is sound, effective, and superior to the existing methods, such as the classical extended Kalman filter and the method of body-fixed representation proposed in [1]. The proposed method does not encounter the problem of divergence associated with the former and converges faster than the latter, with about $20 \%$ reduction of steady-state errors.

\section{Constrained filtering method}

\subsection{Problem description: nonlinear system with constraints}

When applying a filtering method to many physical problems, such as attitude determination, nonlinear effects with extra constraints frequently arise. Consider a nonlinear discrete dynamical system described by the state vector $\boldsymbol{x}_{k} \in \mathbb{R}^{n}$ with the cvolution equation

$$
\boldsymbol{x}_{k+1}=\boldsymbol{f}_{k}\left(\boldsymbol{x}_{k}\right)+\boldsymbol{\zeta}_{k}
$$


where $f_{k}$ denotes a nonlinear map from $\mathbb{R}^{n}$ to $\mathbb{R}^{n}$ and $\zeta_{k}$ represents the process noise with zero mean and covariance matrix $Q_{k}$. If the system is further constrained by

$$
l_{k}\left(x_{k}\right)=0
$$

where $l_{k}$ is the nonlinear constraint function mapping $\mathbb{R}^{n}$ to $\mathbb{R}^{\prime}$, the corresponding filtering problcm is to find an estimate of the state from the measurement vector $z_{k} \in \mathbb{R}^{\prime \prime}$ in the form

$$
z_{k}=\boldsymbol{\Psi}_{k}\left(\boldsymbol{x}_{k}\right)+v_{k}
$$

in which $\boldsymbol{\Psi}_{k}$ denotes a nonlinear map and $v_{k}$ is the measurement noise with zero mean and covariance matrix $\boldsymbol{S}_{k}$, independent of $\boldsymbol{\zeta}_{k}$.

The estimation problem, starting with the prior estimate $\hat{\boldsymbol{x}}_{0}$ and the initial state error covariance matrix $\boldsymbol{P}_{0}$, can be formulated as follows: based on the previous unbiased estimate $\hat{\boldsymbol{x}}_{k}$ with error covariance matrix $\boldsymbol{P}_{k}$ at each step $k=0,1,2, \ldots$, it is desired to find the estimate $\hat{\boldsymbol{x}}_{k-1-1}$ utilising the new measurement $z_{k+1}$ and satisfying the constraint condition (2). For lincar systems, this problem has been solved in [2] by integrating the evolution equation and constraint equation as a linear descriptor system, and the techniques developed for linear singular system in $[2,6,7]$ can be applied. For nonlinear problems, the idea of treating the constraint equation as pseudo-measurements with noise has been adopted in $[8,9]$ by linearising the constraint equation. The method presented in this paper extends the idea of [2] to deal with nonlinear problems, with the nonlinear constraint conditions being resolved exactly.

Define the state error vector $\gamma_{k}=x_{k}-\hat{\boldsymbol{x}}_{k}$. It is assumed that $\gamma_{k}$ is a random vector with zero mean and covariance matrix $\boldsymbol{P}_{k}$, and is independent of $\boldsymbol{\zeta}_{k}$. Furthermore, since the estimation error at step $k$ is determined by the process noise prior to step $k$ and the measurement noise at step $k$, it is reasonable to assume that $\gamma_{k}$ is independent of $\boldsymbol{x}_{k+1}$. With $\gamma_{k}$, the $f_{k}\left(x_{k}\right)$ in (1) can be approximated, up to first order, as

$$
\boldsymbol{f}_{k}\left(\boldsymbol{x}_{k}\right) \cong \boldsymbol{f}_{k}\left(\hat{\boldsymbol{x}}_{k}\right)+\boldsymbol{F}_{k} \gamma_{k} \quad \boldsymbol{F}_{k} \equiv \frac{\partial \boldsymbol{f}_{k}}{\partial \boldsymbol{x}_{k}}\left(\hat{\boldsymbol{x}}_{k}\right)
$$

and (1) is rewritten as

$$
\boldsymbol{f}_{k}\left(\hat{\boldsymbol{x}}_{k}\right)=\boldsymbol{x}_{k+1}-\boldsymbol{F}_{k} \gamma_{k} \dot{-} \zeta_{k}
$$

This equation may be regarded as a pseudo-measurement equation of $\boldsymbol{x}_{k+1}$, with 'measurement' $\boldsymbol{f}_{k}\left(\hat{\boldsymbol{x}}_{k}\right)$, and 'noise' $-\boldsymbol{F}_{k} \boldsymbol{\gamma}_{k}-\boldsymbol{\zeta}_{k}$ with covariance matrix $\boldsymbol{F}_{k} \boldsymbol{P}_{k} \boldsymbol{F}_{k}+\boldsymbol{Q}_{k}$.

On the other hand, the constraint (2) can also be treated as pseudo-mcasurement equation, with 'measurement' zcro, and no 'noise'. Combining all the measurements and pscudo-measurements for state $\boldsymbol{x}_{k+1},(5),(3)$ and (2) are integrated in the following form:

$$
y=h(x)+\xi
$$

where

$$
\begin{aligned}
& \boldsymbol{y}=\left[\begin{array}{c}
\boldsymbol{f}_{k}\left(\hat{\boldsymbol{x}}_{k}\right) \\
\boldsymbol{z}_{k+1} \\
0
\end{array}\right] \quad \boldsymbol{h}\left(\boldsymbol{x}_{k+1}\right)=\left[\begin{array}{c}
\boldsymbol{x}_{k+1} \\
\boldsymbol{\Psi}_{k+1}\left(\boldsymbol{x}_{k+1}\right) \\
\boldsymbol{l}_{k+1}\left(\boldsymbol{x}_{k+1}\right)
\end{array}\right] \text { and } \\
& \boldsymbol{\xi}=\left[\begin{array}{c}
-\boldsymbol{F}_{k} \gamma_{k}-\boldsymbol{\zeta}_{k} \\
v_{k+1} \\
0
\end{array}\right]
\end{aligned}
$$

The corresponding covariance matrix of the disturbances is found to be

$$
\boldsymbol{R}_{k+1}=\left[\begin{array}{ccc}
\boldsymbol{F}_{k} \boldsymbol{P}_{k} \boldsymbol{F}_{k}^{T}+\boldsymbol{Q}_{k} & \mathbf{0} & \mathbf{0} \\
\mathbf{0} & \boldsymbol{S}_{k+1} & \mathbf{0} \\
\mathbf{0} & \mathbf{0} & \mathbf{0}
\end{array}\right]
$$

and is seen to be singular. Such a singularity problem causes the classical maximum-likelihood method to fail, and the extended Kalman filter algorithm is unable to converge. To handle this problem, an extended maximumlikelihood method for estimating $\boldsymbol{x}_{k+1}$ and the associated error covariance matrix is devcloped in the next subsection.

\subsection{Extended maximum-likelihood method}

The classical maximum-likelihood method is to find the optimal estimate of $\boldsymbol{x}$ from the measurement cquation, linear in $\boldsymbol{x}$,

$$
\boldsymbol{y}=\boldsymbol{H} \boldsymbol{x}+\boldsymbol{\xi}
$$

such that the cost function

$$
J=\frac{1}{2} \xi^{T} \boldsymbol{R}^{-1} \xi
$$

is minimised, where $R$ denotes the positive-definite covariance matrix of the noise $\xi$. If now the error covariance matrix $\boldsymbol{R}$ is singular, or positive semi-definite, the classical maximum-likelihood method must be modified. Let $\boldsymbol{\Gamma}$ be the full-column-rank squarc root of $\boldsymbol{R}$ such that $\boldsymbol{R}=\boldsymbol{\Gamma} \boldsymbol{T}^{T}$. The noise $\xi$ may be generated by a zero-mean random vector $\eta$, with identity covariance matrix, such that $\boldsymbol{\xi}=\boldsymbol{\Gamma} \boldsymbol{\eta}$. Furthermore, if the problem is nonlinear, (9) may be rewritten as

$$
y=h(x)+\xi=h(x)+\Gamma \eta
$$

The associated estimation problem is now cast to be the problem of minimising

$$
J=\frac{1}{2} \boldsymbol{\eta}^{f^{\prime}} \boldsymbol{\eta}
$$

subject to the nonlinear constraint ( 11 ) on $\boldsymbol{x}$ and $\boldsymbol{\eta}$

With the Lagrange multiplier vector $\boldsymbol{\lambda}$, the previous problem can be solved by finding the critical condition of the Lagrangian

$$
L(\boldsymbol{\eta}, \boldsymbol{x}, \boldsymbol{\lambda})=\frac{1}{2} \boldsymbol{\eta}^{T} \boldsymbol{\eta}+\boldsymbol{\lambda}^{T}(\boldsymbol{y}-\boldsymbol{h}(\boldsymbol{x})-\boldsymbol{\Gamma} \boldsymbol{\eta})
$$

which gives rise to the constraint condition (11) and the following equations

$$
\begin{aligned}
\boldsymbol{\eta}-\boldsymbol{\Gamma}^{T} \boldsymbol{\lambda} & =0 \\
\boldsymbol{H}(\boldsymbol{x})^{T} \boldsymbol{\lambda} & =0
\end{aligned}
$$

where $\boldsymbol{H}(\boldsymbol{x})=\partial \boldsymbol{h} / \partial \boldsymbol{x}(\boldsymbol{x})$. From (14) and (11), we obtain

$$
h(x)+R \lambda=y
$$

The optimal estimate is then obtained by solving the previous equation and (15) simultaneously for $\boldsymbol{x}$ and $\boldsymbol{\lambda}$.

With the given prior estimate $\boldsymbol{x}^{-}$, the set of equations (15), (16) may be solved by using the Newton-Raphson iterative method. First, the initial condition is obtained by solving the linearised equations of (15), (16) about $\boldsymbol{x}^{-}$,

$$
\begin{aligned}
\boldsymbol{H}\left(\boldsymbol{x}^{-}\right)^{T} \boldsymbol{\lambda} & =0 \\
\boldsymbol{H}\left(\boldsymbol{x}^{-}\right) \boldsymbol{x}+\boldsymbol{R} \boldsymbol{\lambda} & =\boldsymbol{y}-\boldsymbol{h}\left(\boldsymbol{x}^{-}\right)+\boldsymbol{H}\left(\boldsymbol{x}^{-}\right) \boldsymbol{x}^{-}
\end{aligned}
$$


In terms of the pseudo-inverse $[10,11]$, the solution is found to be

$$
\begin{aligned}
{\left[\begin{array}{l}
\boldsymbol{\lambda}_{0} \\
\boldsymbol{x}_{0}
\end{array}\right]=} & {\left[\begin{array}{cc}
\boldsymbol{R} & \boldsymbol{H}\left(\boldsymbol{x}^{-}\right) \\
\boldsymbol{H}^{T}\left(\boldsymbol{x}^{-}\right) & 0
\end{array}\right]^{+} } \\
& \times\left[\begin{array}{c}
\boldsymbol{y}-\boldsymbol{h}\left(\boldsymbol{x}^{-}\right)+\boldsymbol{H}\left(\boldsymbol{x}^{-}\right) \boldsymbol{x}^{-} \\
\mathbf{0}
\end{array}\right]
\end{aligned}
$$

The corresponding initial crror covariance matrix $\boldsymbol{P}_{0}$ for $\boldsymbol{x}_{0}$ is given by

$$
\boldsymbol{P}_{0}=-\left[\begin{array}{ll}
0 & \boldsymbol{I}
\end{array}\right]\left[\begin{array}{cc}
\boldsymbol{R} & \boldsymbol{H}\left(\boldsymbol{x}^{-}\right) \\
\boldsymbol{H}\left(\boldsymbol{x}^{-}\right)^{T} & \mathbf{0}
\end{array}\right]^{+}\left[\begin{array}{l}
\mathbf{0} \\
\boldsymbol{I}
\end{array}\right]
$$

The iteration to find the solution of (15), (16) then proceeds as follows, for $j=0,1,2, \ldots$ :

$$
\left[\begin{array}{c}
\lambda_{j+1} \\
\boldsymbol{x}_{j+1}
\end{array}\right]=\left[\begin{array}{c}
\lambda_{j} \\
\boldsymbol{x}_{j}
\end{array}\right]+\boldsymbol{W}_{j}^{-1}\left[\begin{array}{c}
-\boldsymbol{H}^{T}\left(\boldsymbol{x}_{j}\right) \boldsymbol{\lambda}_{j} \\
-\boldsymbol{h}\left(\boldsymbol{x}_{j}\right)-\boldsymbol{R} \boldsymbol{\lambda}_{j}+\boldsymbol{y}
\end{array}\right]
$$

where

$$
\boldsymbol{W}_{j}=\left[\begin{array}{cc}
\boldsymbol{H}^{T}\left(\boldsymbol{x}_{j}\right) & \left.\left(\frac{\partial}{\partial \boldsymbol{x}} \boldsymbol{H}^{T}(\boldsymbol{x})\right) \boldsymbol{\lambda}\right|_{\left(\boldsymbol{\lambda}_{j}, \boldsymbol{x}_{j}\right)} \\
\boldsymbol{R} & \boldsymbol{H}\left(\boldsymbol{x}_{j}\right)
\end{array}\right]
$$

Once the iterations converge at step $j+1$, the maximumlikelihood estimate $\hat{\boldsymbol{x}}$ is given by $\boldsymbol{x}_{j+1}$. The corresponding error covariance matrix for $\hat{\boldsymbol{x}}$ is derived by performing the following iterative process,

$$
\begin{aligned}
\boldsymbol{P}_{j+1} & \equiv E\left[\left(\boldsymbol{x}-\boldsymbol{x}_{j+1}\right)\left(\boldsymbol{x}-\boldsymbol{x}_{j+1}\right)^{T}\right] \\
& =\boldsymbol{P}_{j}-\boldsymbol{P}_{1, j}-\boldsymbol{P}_{1, j}^{T}+\boldsymbol{P}_{2, j}
\end{aligned}
$$

where

$$
\begin{aligned}
\boldsymbol{P}_{\mathrm{I}, j} & =E\left[\left(\boldsymbol{x}_{j+1}-\boldsymbol{x}_{j}\right)\left(\boldsymbol{x}-\boldsymbol{x}_{j}\right)^{T}\right] \\
& =\left[\begin{array}{ll}
0 & \boldsymbol{I}
\end{array}\right] \boldsymbol{W}_{j}^{-\cdot 1}\left[\begin{array}{c}
\mathbf{0} \\
\boldsymbol{H}\left(\boldsymbol{x}_{j}\right) \boldsymbol{P}_{j}
\end{array}\right]
\end{aligned}
$$

and

$$
\begin{gathered}
\boldsymbol{P}_{2, j}=E\left[\left(\boldsymbol{x}_{j+1}-\boldsymbol{x}_{j}\right)\left(\boldsymbol{x}_{j+1}-\boldsymbol{x}_{j}\right)^{T}\right]=\left[\begin{array}{ll}
0 & \boldsymbol{I}
\end{array}\right] \boldsymbol{W}_{j}^{-1} \\
{\left[\begin{array}{cr}
\boldsymbol{H}^{T}\left(\boldsymbol{x}_{j}\right) \boldsymbol{\lambda}_{j} \boldsymbol{\lambda}_{j}^{T} \boldsymbol{H}\left(\boldsymbol{x}_{j}\right) & \boldsymbol{H}^{T}\left(\boldsymbol{x}_{j}\right) \boldsymbol{\lambda}_{j} \boldsymbol{\lambda}_{j}^{T} \boldsymbol{R}^{T} \\
\boldsymbol{R} \boldsymbol{\lambda}_{j} \boldsymbol{\lambda}_{j}^{T} \boldsymbol{H}\left(\boldsymbol{x}_{j}\right) & \boldsymbol{H}\left(\boldsymbol{x}_{j}\right) \boldsymbol{P}_{j} \boldsymbol{H}^{T}\left(\boldsymbol{x}_{j}\right)+\boldsymbol{R} \\
& +\boldsymbol{R} \boldsymbol{\lambda}_{j} \boldsymbol{\lambda}_{j}^{T} \boldsymbol{R}^{T^{T}}
\end{array}\right]} \\
\times \boldsymbol{W}_{j}^{-T}\left[\begin{array}{l}
\mathbf{0} \\
\boldsymbol{I}
\end{array}\right]
\end{gathered}
$$

With the prior estimate suitably chosen, the estimate $\hat{\boldsymbol{x}}$, as well as the error covariance matrix $\hat{\boldsymbol{P}}$, can be obtained within steps in the Newton-Raphson iteration.

Simply by substituting the associated terms in (7) with the 'noise' covariance matrix given by (8), the abovedeveloped extended maximum-likelihood method can be used to solve the problem formulated in the previous subsection. The iterative method in finding the estimate $\boldsymbol{x}_{k+1}$ with each step applying the extended maximumlikelihood method is termed the constrained filtering method, as depicted in Fig. 1. The prior error covariance matrix $\boldsymbol{P}_{k}$ enters through (8) and (20) to determine the initial value of the iterative process (23). Since at each step of the filter, the previous estimate is used as the prior estimate for the next step, the convergence of the NewtonRaphson itcration can be attained.

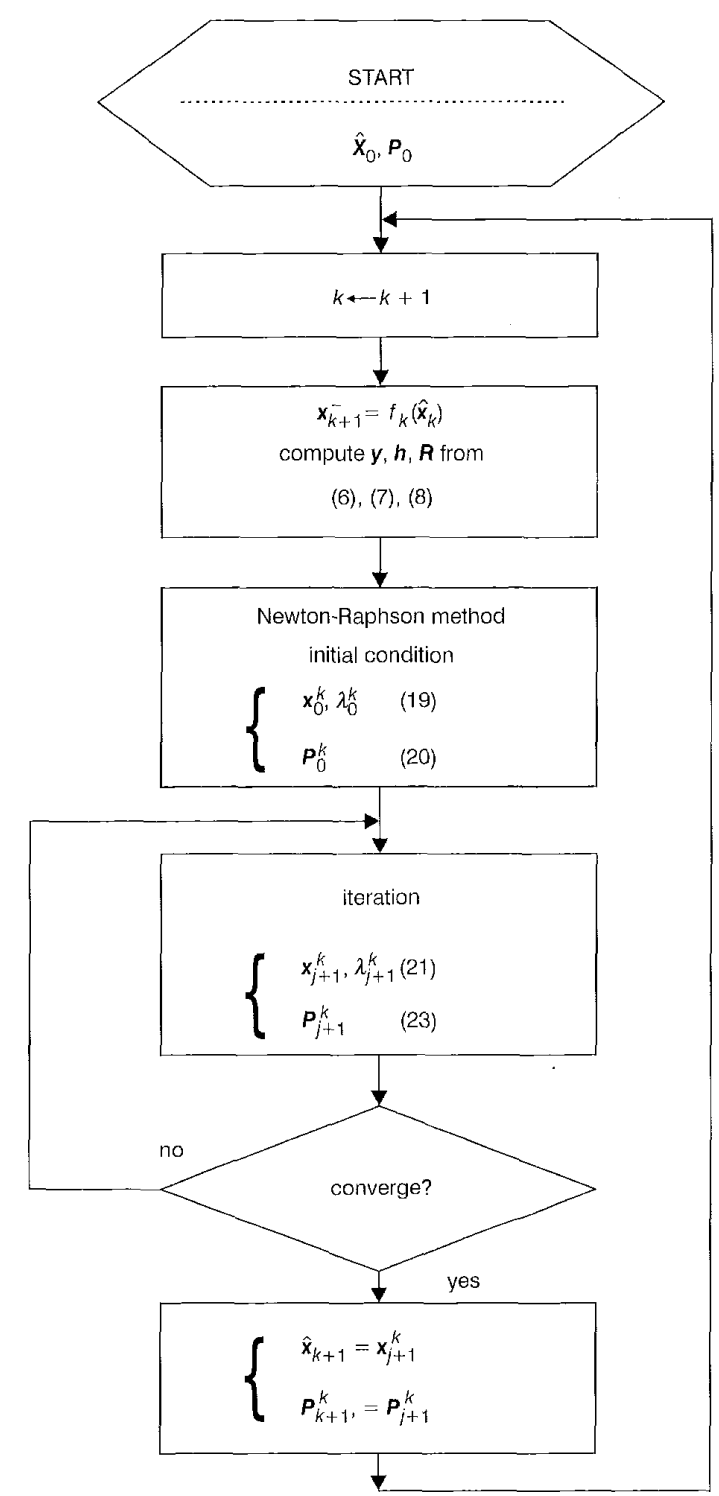

Fig. 1 Flowchart of constrained filtering method

\section{Attitude determination algorithm}

The constrained filtering method for the nonlinear system developed in the previous section is now applied to deal with the unit norm constraint associated with attitude detcrmination problems. The attitude motion of a vehicle may be described by a time-varying $3 \times 3$ rotation or attitude matrix $\boldsymbol{A}(t)$ such that $\boldsymbol{A}(t)^{T} \boldsymbol{A}(t)=\boldsymbol{I}$, det $\boldsymbol{A}(t)=1$. Among those representations for the attitude, such as Eulerian angles [12], the unit quaternion $\bar{q}$ with components $\left(q_{1}, q_{2}, q_{3}, q_{4}\right)$ satisfying the unit norm condition,

$$
q_{1}^{2}+q_{2}^{2}+q_{3}^{2}+q_{4}^{2}=1
$$

provides the global representation with the least number of parameters. Let $\omega=\left[\begin{array}{lll}\omega_{1} & \omega_{2} & \omega_{3}\end{array}\right]^{7}$ denote the angular velocity of the body. The rate of change of the unit quaternion can be expressed as

$$
\frac{d}{d t} \bar{q}(t)=\frac{1}{2} \Omega(\omega) \bar{q}(t)=\frac{1}{2} \Xi(\ddot{q}) \omega(t)
$$


where

$$
\begin{aligned}
& \boldsymbol{\Omega}(\boldsymbol{\omega})=\left[\begin{array}{cccc}
0 & \omega_{3} & -\omega_{2} & \omega_{1} \\
-\omega_{3} & 0 & \omega_{1} & \omega_{2} \\
\omega_{2} & -\omega_{1} & 0 & \omega_{3} \\
-\omega_{1} & -\omega_{2} & -\omega_{3} & 0
\end{array}\right] \\
& \boldsymbol{\Xi}(\ddot{\boldsymbol{q}})=\left[\begin{array}{ccc}
q_{4} & -q_{3} & q_{2} \\
q_{3} & q_{4} & -q_{1} \\
-q_{2} & q_{1} & q_{4} \\
-q_{1} & -q_{2} & -q_{3}
\end{array}\right]
\end{aligned}
$$

Here, (28) serves as the evolution equation to perform attitude determination, if the angular velocity is known. To measure the angular velocity, a rate gyro may be adopted, whose model in given in the next subsection.

\subsection{Gyro model}

A simple but realistic model of the rate gyro can be found in $[13,12]$, in which the gyro output vector $\boldsymbol{u}$ is related to the angular velocity $\omega$ by

$$
\boldsymbol{u}=\boldsymbol{a}+\boldsymbol{b}+\boldsymbol{\eta}_{1}
$$

where $\boldsymbol{\eta}_{1}$ is a zero-mean white Gaussian noise with strength $\boldsymbol{Q}_{1}$ and $\boldsymbol{b}$ denotes the gyro drift, which is assumed to be governed by

$$
\frac{d}{d t} \boldsymbol{b}=\boldsymbol{\eta}_{2}
$$

where $\boldsymbol{\eta}_{2}$ is an independent zero-mean Gaussian noise with strength $\boldsymbol{Q}_{2}$. The gyro measurement is useful for long duration only if the effect of the drift $b$ can be properly compensated. Hence, the previous dynamical equation shall be included in the system for the proposed attitude determination algorithm to obtain an estimate of $\boldsymbol{b}$.

\subsection{State equations}

For the attitude determination problem with quaternion representation and the measurements from the gyros, the state vector is chosen as

$$
x=\left[\begin{array}{l}
\bar{q} \\
b
\end{array}\right]
$$

From (30), then (28) can be rewritten as

$$
\begin{aligned}
\dot{\bar{q}} & =\frac{1}{2} \boldsymbol{\Omega}\left(\boldsymbol{u}-\boldsymbol{b}-\boldsymbol{\eta}_{1}\right) \overline{\boldsymbol{q}} \\
& =\frac{1}{2} \boldsymbol{\Omega}(\boldsymbol{u}-\boldsymbol{b}) \overline{\boldsymbol{q}}-\frac{1}{2} \boldsymbol{\Xi}(\overline{\boldsymbol{q}}) \boldsymbol{\eta}_{1}
\end{aligned}
$$

Note that the drift vector is a random walk, which means, in a discrete sense, that it should remain a constant vector, i.e. $\boldsymbol{b}_{k}$, during the time interval $\left(t_{k}, t_{k+1}\right)$. The previous equation can then be expressed as

$$
\dot{\overline{\boldsymbol{q}}}=\frac{1}{2} \boldsymbol{\Omega}\left(\boldsymbol{u}-\boldsymbol{b}_{k}\right) \overline{\boldsymbol{q}}-\frac{1}{2} \boldsymbol{\Xi}(\overline{\boldsymbol{q}}) \boldsymbol{\eta}_{1} \quad t \in\left(t_{k}, t_{k+1}\right)
$$

Furthermore, it is observed that the expectation of the second term in the right-hand side vanishes due to the assumption of zero-mean for $\boldsymbol{\eta}_{1}$. The variable $\bar{q}$ in that term can be thus approximated by $\bar{q}_{k}$, since it affects mainly the strength of the random noise. The simplified version of (34),

$$
\dot{\bar{q}}=\frac{1}{2} \boldsymbol{\Omega}\left(\boldsymbol{u}-\boldsymbol{b}_{k}\right) \overline{\boldsymbol{q}}-\frac{1}{2} \boldsymbol{\Xi}\left(\overline{\boldsymbol{q}}_{k}\right) \boldsymbol{\eta}_{1} \quad t \in\left(t_{k}, t_{k+1}\right)
$$

is now a time-varying linear dynamical equation for $\overline{\boldsymbol{q}}$.
Equations (35) and (31) provide the evolution equations for the state vector $\boldsymbol{x}$ in $\left(t_{k}, t_{k+1}\right)$. They can be discretised as

$$
\boldsymbol{x}_{k+1}=\boldsymbol{f}_{k}\left(\boldsymbol{x}_{k}\right)+\boldsymbol{\zeta}_{k}
$$

where, in terms of the matrix exponential exp,

$$
\boldsymbol{f}_{k}\left(\boldsymbol{x}_{k}\right)=\left[\begin{array}{c}
\exp \left(\frac{1}{2} \int_{t_{k}}^{t_{k+1}} \boldsymbol{\Omega}\left(\boldsymbol{u}(\tau)-\boldsymbol{b}_{k}\right) d \tau\right) \overline{\boldsymbol{q}}_{k} \\
\boldsymbol{b}_{k}
\end{array}\right]
$$

and $\zeta_{k}$ is given by

$$
\boldsymbol{\zeta}_{k}=\int_{t_{k}}^{t_{k+1}} \exp \left(\int_{\tau}^{t_{k+1}} \boldsymbol{F}_{k}(\sigma) d \sigma\right) \boldsymbol{G}_{k}\left[\begin{array}{l}
\boldsymbol{\eta}_{1}(\tau) \\
\boldsymbol{\eta}_{2}(\tau)
\end{array}\right] d \tau
$$

in which

$$
\begin{aligned}
\boldsymbol{F}_{k}(\sigma) & =\frac{\partial \boldsymbol{f}_{k}}{\partial \boldsymbol{x}_{k}}\left(\boldsymbol{x}_{k}\right)=\left[\begin{array}{cc}
\frac{1}{2} \boldsymbol{\Omega}\left(\boldsymbol{u}(\sigma)-\boldsymbol{b}_{k}\right) & \mathbf{0} \\
\mathbf{0} & \mathbf{0}
\end{array}\right]_{7 \times 7} \\
\boldsymbol{G}_{k} & =\left[\begin{array}{cc}
-\frac{1}{2} \boldsymbol{\Xi}\left(\overrightarrow{\boldsymbol{q}}_{k}\right) & \mathbf{0} \\
\mathbf{0} & \boldsymbol{I}
\end{array}\right]_{7 \times 6}
\end{aligned}
$$

In addition, recall that the covariance matrix for $\left(\boldsymbol{\eta}_{1}, \boldsymbol{\eta}_{2}\right)$ is

$$
\boldsymbol{Q}=\left[\begin{array}{cc}
Q_{1} & \mathbf{0} \\
\mathbf{0} & Q_{2}
\end{array}\right]
$$

The disturbance $\zeta_{k}$ is then found to be a zero-mean Gaussian noise with covariance matrix

$$
\begin{aligned}
\boldsymbol{Q}_{k}= & \int_{t_{k}}^{t_{k+1}} \exp \left(\int_{\tau}^{t_{k+1}} \boldsymbol{F}_{k}(\sigma) d \sigma\right) \boldsymbol{G}_{k} \\
& \times \boldsymbol{Q} \boldsymbol{G}_{k}^{T} \exp \left(\int_{\tau}^{t_{k+1}} \boldsymbol{F}_{k}(\sigma) d \sigma\right)^{T} d \tau
\end{aligned}
$$

Due to the presence of gyro drift, it is necessary to utilise other measurements to complete the process of attitude determination. Among various attitude sensors, such as the star tracker, the magnetometer, and GPS, ctc., the emerging technology of GPS attitude determination is employed, and this is now discussed.

\subsection{Measurement equation and filtering method}

With accurate carrier phase observables, it is possible to perform attitude determination using GPS as discussed in [14-17]. Consider a platform with $\alpha(\geq 2)$ baseline vectors represented, respectively, by known vectors $\boldsymbol{a}_{i}, i=1, \ldots, \alpha$ in the body frame. At each end of every baseline, one GPS antenna is mounted, and it is assumed that there are $\beta(\geq 4)$ GPS satellites in sight, each with a unit direction vector $\boldsymbol{g}^{j}$. Define $\boldsymbol{s}^{j}=\boldsymbol{g}^{j+1}-\boldsymbol{g}^{1}, j=1, \ldots, \beta-1$. Following the method used in [18], the double difference carricr phase observables $\psi_{i}^{j}\left(t_{k}\right)$ for baseline $i$ can be expressed as

$$
\psi_{i}^{j}\left(t_{k}\right)=\overline{\boldsymbol{q}}^{\top}\left(t_{k}\right) \boldsymbol{K}_{i j}\left(t_{k}\right) \overline{\boldsymbol{q}}\left(t_{k}\right)+v_{i}^{j}\left(t_{k}\right)
$$

where the $4 \times 4$ matrix $\boldsymbol{K}_{i j}$ is given by

$$
\boldsymbol{K}_{i j}=\left[\begin{array}{cc}
\boldsymbol{a}_{i}\left(\boldsymbol{s}^{j}\right)^{T}+\boldsymbol{s}^{j} \boldsymbol{a}_{i}^{T}-\left(\boldsymbol{a}_{i}^{T} \boldsymbol{s}^{j}\right) \boldsymbol{I} & \boldsymbol{a}_{i} \times \mathbf{s}^{j} \\
{\left[\boldsymbol{a}_{i} \times \mathbf{s}^{j}\right]^{T}} & \boldsymbol{a}_{i}^{T} \mathbf{s}^{j}
\end{array}\right]
$$

and $v_{j}^{j}$ is assumed to be a white noise with distribution $\mathcal{N}\left(0, S_{i}^{j}\right)$. 
Combining all the measurement equations, with total number of $\alpha(\beta-1)$, the matrix form of the measurement equations can be expressed as

$$
\begin{aligned}
{\left[\begin{array}{c}
\psi_{1}^{1} \\
\psi_{1}^{2} \\
\vdots \\
\psi_{\alpha}^{\beta-1}
\end{array}\right]=} & {\left[\begin{array}{cccc}
\overline{\boldsymbol{q}}^{T} & 0 & \ldots & 0 \\
0 & \overline{\boldsymbol{q}}^{T} & \ldots & 0 \\
\vdots & \vdots & \ddots & \vdots \\
0 & 0 & \ldots & \overline{\boldsymbol{q}}^{T}
\end{array}\right] } \\
& \times\left[\begin{array}{c}
\boldsymbol{K}_{11} \\
\boldsymbol{K}_{12} \\
\vdots \\
\boldsymbol{K}_{\alpha(\beta-1)}
\end{array}\right] \overline{\boldsymbol{q}}+\left[\begin{array}{c}
v_{1}^{1} \\
v_{1}^{2} \\
\vdots \\
v_{\alpha}^{\beta-1}
\end{array}\right]
\end{aligned}
$$

Wo are now ready to design the filter for attitude determination using gyro outputs and GPS observables. The problem falls into the category of nonlinear constrained filtering problem discussed in Section 2.1, with the evolution equation (36), the nonlinear constraint in the form of (2) where

$$
l(x)=q_{1}^{2}+q_{2}^{2}+q_{3}^{2}+q_{4}^{2}-1
$$

and the nonlincar measurement equation (44). The method developed in Section 2.2 can then be adopted to design an optimal filter. As will be seen in the next section, the proposed filter can solve the attitude determination problem effectively.

\section{Scenario and simulation results}

\subsection{Scenario setup}

To assess the performance of the proposed filtering method, Monte-Carlo simulations with appropriate models and scenario were conducted. For the simulation, the configuration of the platform was as shown in Fig. 2, with gyro mounted at $D$ and three GPS antenna mounted at $R_{A}, R_{B 3}, R_{C}$, respectively, to form two baseline vectors expressed in the body frame as

$$
\begin{aligned}
& \boldsymbol{a}_{1}=R_{A} R_{B}=1.325\left[\begin{array}{lll}
1 & 0 & 0
\end{array}\right] \\
& a_{2}=R_{A} R_{C}=1.445\left[\cos \left(62.71^{\circ}\right) \quad \sin \left(62.71^{\circ}\right) 0\right]
\end{aligned}
$$

Assume that during the process, seven satellites with PRN numbers $4,6,8,10,24,26$ and 27 can be observed, with GDOP being 2.0. The positions of these satellites can be computed from the ephemeris data, and ambiguity-free GPS phase observables can be generated by the simulator,

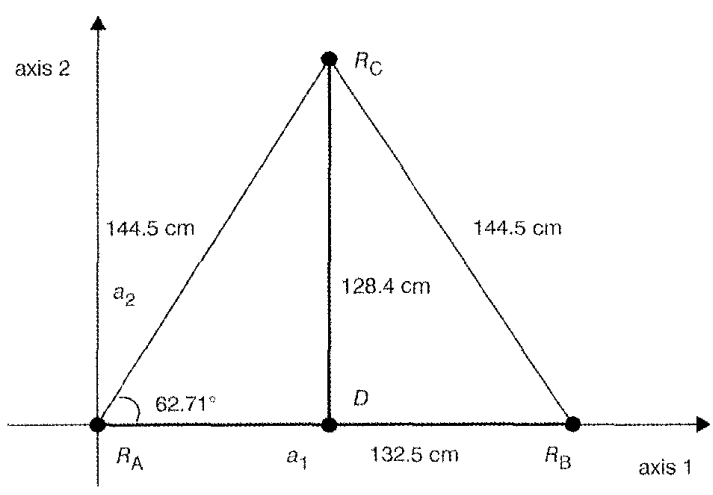

Fig. 2 Configuration of two baseline vectors for simulations and experiments

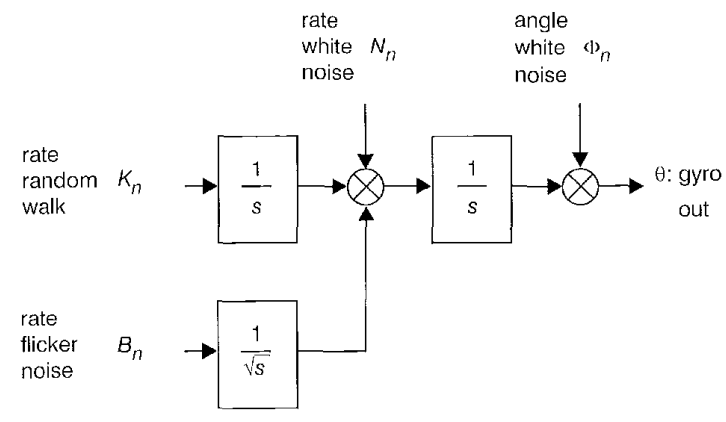

Fig. 3 Block diagram of stochastic model of gyro noise

with white noises $\mathcal{N}\left(0,(0.01 \lambda)^{2}\right)$ being imposed, where $\lambda$ denotes the wavelength of the carricr.

While the simple model for gyro output (30) is used to design the filter, a more realistic model is adopted to generate gyro measurements in the simulator. It is assumed that the rate-integrated laser-ring gyro produced by Litton (SIRU) is chosen. According to [19], the gyro noise model is shown in Fig. 3 with parameters listed in Table. 1.

\subsection{Simulation results and comparison with other existing methods}

To perform the software simulation, it is assumed that the platform is rotated about $D$ with constant angular velocity $\left[\begin{array}{lll}0 & -1 \times 10^{-3} & 0\end{array}\right]^{T} \mathrm{rad} / \mathrm{s}$, with the initial attitude given by the quaternion $\overline{\boldsymbol{q}}_{i n i}=\left[\begin{array}{llll}0 & 1 & 0 & 0\end{array}\right]^{T}$. With the gyro model of Litton (SIRU) and (GPS LI carrier phase model used in the simulator, the filtering method discussed in Section 3 is applied to determine the 3-D attitude motion. In terms of the roll-pitch-yaw angles, the steady-state errors from one Monte-Carlo simulation are shown in Fig. 4. The corresponding $3 \sigma$ values are listed in the second row of Table. 2.

To assess the performance of the proposed scheme, the classical extended Kalman filter was first applied to the same scenario. The trace of the covariance matrix using the proposed filter and the extended Kalman filter during the initial 240 seconds are shown in Fig. 5. While the extended Kalman filter converges in the first few steps, it diverges after 150 seconds due to the accumulation of round-off crrors, since the corresponding covariance matrix becomes singular. Ignoring the constraint condition renders the classical extended Kalman filter method invalid for attitude determination.

The method of body-fixed covariance representation discussed in $[1,20,21]$ was also used to estimate the

Table 1: Gyro error characteristics

\begin{tabular}{lcc}
\hline $\begin{array}{l}\text { Gyro } \\
\text { parameters }\end{array}$ & Value & Comment \\
\hline Noise & rate flicker error & IEEE standard \\
& $B_{n}: 0.002$ deg $/ \mathrm{h}$ & definition [13] \\
& rate white noise \\
& $N_{n}: 0.0006 \mathrm{deg} / \mathrm{h}$ & \\
& angle white noise & \\
& $\Phi_{n}: 0.019^{\prime \prime} / \mathrm{h}$ & \\
& rate random walk & \\
& $K_{n}: 0$ & \\
& 3 deg $/ \mathrm{h}(3 \sigma)$ & \\
Constant & & \\
drift & & \\
\hline
\end{tabular}



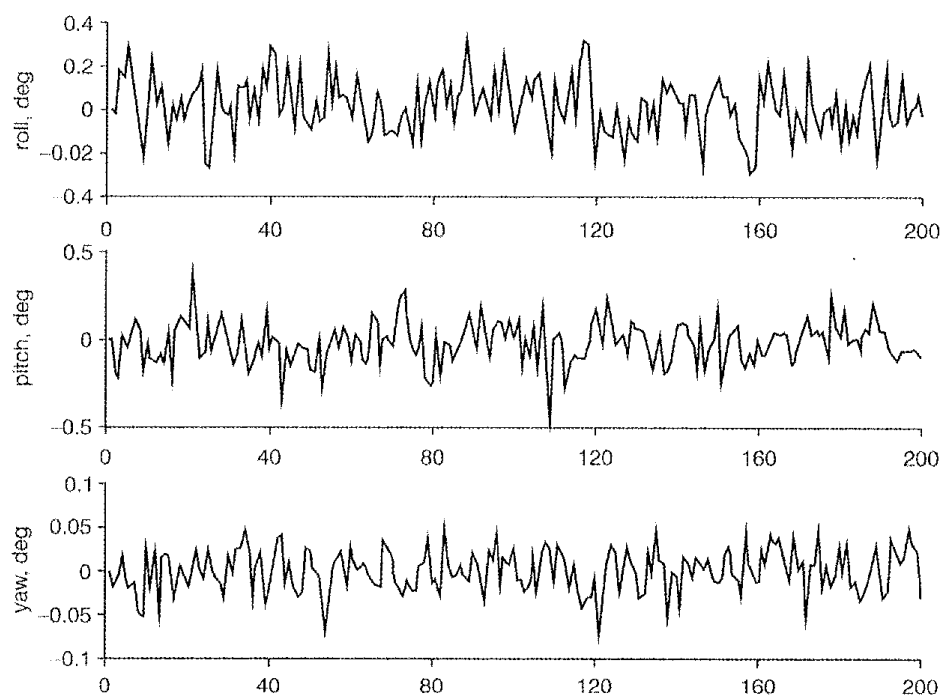

Fig. 4 Steady-state estimation errors using the constrained fittering method

Table 2: Accuracies of constrained filtering method and body-fixed covariance representation method

\begin{tabular}{llll}
\hline Steady state errors & $3 \sigma_{\text {roll }}(\mathrm{deg})$ & $3 \sigma_{\text {pitch }}(\mathrm{deg})$ & $3 \sigma_{\text {yaw }}(\mathrm{deg})$ \\
\hline $\begin{array}{c}\text { Constrained filtering } \\
\text { method }\end{array}$ & 0.3733 & 0.3609 & 0.0727 \\
$\begin{array}{c}\text { Body-fixed covariance } \\
\text { representation method }\end{array}$ & 0.4899 & 0.4761 & 0.0985 \\
\hline
\end{tabular}

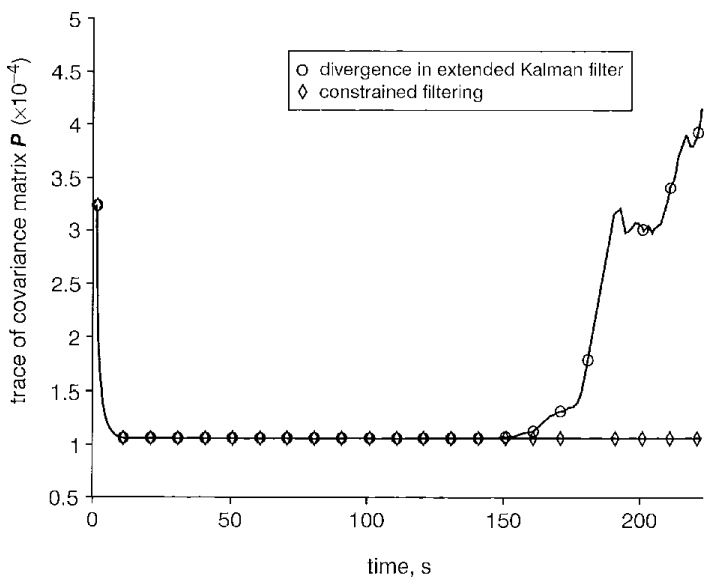

Fig. 5 Evolutions of the trace of the covariance matrix using the methods of constrained filtering and the extended Kalman filter

attitude for exactly the same problem. The algorithm converges and the singularity problem disappcars. While the pattern of the steady-state crror is almost the same as that shown in Fig. 4, it is observed that the errors as listed in the third row of Table 2 are more than $20 \%$ higher than those with the constrained filtering method. On the other hand, both methods were employed to conduct attitude determination with initial condition that the pitch error is set to $30^{\circ}$. The estimated pitch angles for the first 40 seconds are shown in Fig. 6, and it is seen that while the method of body-fixed covariance representation requires

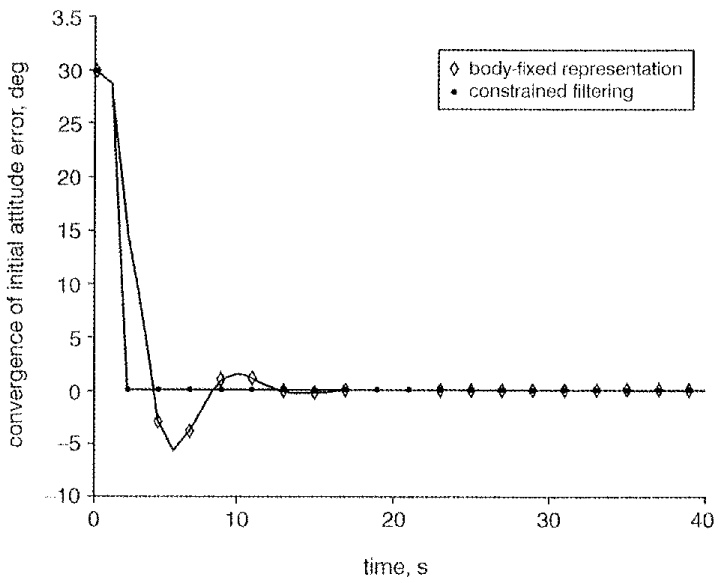

Fig. 6 Comparison of convergences using the constrained fittering method and the method of body-fixed representation

about 15 seconds to converge, the scheme proposed in this paper needs only 2 seconds. Accordingly, from the point of view of either steady-state error or the convergence rate, the constrained filtering method performs better than the method of body-fixed representation for attitude determination.

\section{Conclusions}

$\Lambda \mathrm{n}$ integrated scheme using GPS carricr phase observables and gyro measurements for attitude determination has been presented. The nonlinear constraint of the unit norm on the quaternion representation of the attitude is handled by the proposed constrained filtering method. Simulation results demonstrate the significance of the proposed methodology. However, although the method adopted here may be superior to others in terms of steady-state errors and convergence rate, it may face the problem of complex computation, due to the inclusion of Lagrange multipliers and the use of the Newton-Raphson method. Accordingly, if it is desired to use the proposed method in real time, one may need to employ a powerful signal processor or find a more efficient method to solve the nonlinear algcbraic 
equation. Once this problem is solved, the constrained filtering method may be applied not only to the attitude determination problem but also to a variety of filtering problems associated with nonlinear systems subject to nonlinear constraints.

\section{References}

1 LI.FFERTS, E.J., MARKLEY, F.L., and SHUSTER, M.D.: 'Kalman filtering for spacecraft attitude estimation', J. Gutid. Control Dyn., 1982, 5, (5), pp. 417-429

2 NIKOUKHAH, R. WILLSKY, A.S., and LEVY, B.C.: 'Kalman filtering and Riccati cquations for descriptor systems', IEEE Trons. Autom. Control, 1992, 37, (9), pp. 1325-1342

3 PENG, II.M., CHIANG, Y.T., CHANG, FR., and WANG, L.S.: "Maximum-likelihood-based filtering for attitude determination via GPS carricr phase'. Proceedings of IEEE Position Location and Navigation Symposium, San Diego, California, March 2000, pp. 480487

4 GLBRE-EGZIABHIR, D., HATYWARD, R.C., and POWELL, J.D "A low-cost GPS/inertial attitude heading reference system (ALtRS) for gencral aviation applications'. Proceedings of IEEE Position Location and Navigation Symposium, San Diego, California, March 1998 , pp. $518-525$

5 HAYWARD, R.C.: 'Inertially aided GPS based attitude heading reference system (AHRS) for general aviation aircraft'. Proceedings of IONGPS-97, Kanas City, MO, September 1997, pp. 289-298

6 CAMPBELL, S.L.: 'Singular systems of differential equations' (Pitman, San Francisco, CA, 1980)

7 CAMPBELL, S.L.: 'Singular systems of differential equations, II' (Pitman, San Francisco, CA, 1982)

8 GEETER, J.D., BRUSSEL, H.V., and SCHUTTER, J.D.: 'A smoothly constrained Kalman filter', IEEE Trans. Pattern. Anal. Mach. Intell., 1997, 19, pp. 1171-1177?
9 TAHK, M., and SPLYER, J.L.: 'Target tracking problems subject to kinematic constraints', IEEE Thans. Autom. Control, 1990, 38 pp. $324-326$

10 CAMPBELL, S.L., and MEYLR, C.D.: 'Generalized inverse of linear transforms' (Pitman, London. 1979)

11 LEON, S.G.: 'Linear algebra with applications' (Macmillan Collego Publishing Company, 1994, 4th edn.)

12 WERTZ, J.R.: 'Spacecraft attitude determination and control' (Kluwer Academic Publishers, 1978)

13 FARRENKOPF, R.L.: 'Analytic steady-state accuracy solutions for two common spacecraft attitude cstimator', J. Guid. Control Dyn., 1978, 1, (4), pp. 282-284

14 COHLiN, C.E.: 'Attitude determination using GPS: development of an al solid-state guidance, navigation, and control sensor for air and space vehicles based on the global positioning system?. PhD dissertation, Stanford University, 1992

15 FUJIKAWA. S.J., and ZIMBELMAN, D.F.: 'Spacecraft attitude determination by Kalman filtering of global positioning system signals', J. Guid. Control Dyn., 1995, 18, (6), pp. 1365-1373

16 LU, G.: "Development of a GPS multi-antenna system for attitude determination'. PhD dissertation, University of Calgary, 1995

17 TU, C.II., TU, K.Y., CHANG, F.R., and WANG, L.S.: 'GPS compass: novel navigation equipment', IEEE Trans: Aerosp. Lilectron. Syst. 1997 33, (3), pp. 1063-1068

18 BAR-ITZHACK, T.Y., MONTGOMTRY, P.Y., and GARRICK. J.C. Algorithms for attitude determination using the global positioning system', J. Guid. Control Dyn., 1998, 21, (6), pp. 846-852

19 -iELE standard specification format guide and test procedure for singleaxis laser gyroscopes'. IEEI: Std. 647, 1995

20 AXELRAD, P., and WARD, L.M.: 'Spacecraft attitude estimation using the global positioning system: methodology and results for RADCAL', f. Guid. Control Dyn., 1996, 19, (6), pp. 1201-1209

21 GAI, E., DALY, K., HARRISON, I., and LFMOS, L.: 'Star-sensor-based satellite attitude/atlitude rate estimators', J. Guid. Control Dyn., 1985, 8 (5), pp. $560-565$ 